\title{
Potencialidade social e econômica da pesca e maricultura no Estado do Espírito Santo, Brasil *
}

\author{
Social and economic potential of fishery and aquaculture at \\ Espirito Santo State, Brazil
}

\author{
J. B. Teixeira ${ }^{1}$, A.C. Lima $^{2}$, F.P. Boechat ${ }^{3}$, R.L. Rodrigues ${ }^{3}$, R. R. Freitas ${ }^{\circledR, 3}$
}

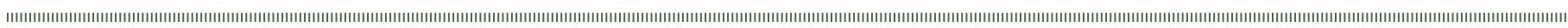

\section{RESUMO}

O estado do Espírito Santo (Brasil) é possui uma extensão litorânea $(\sim 410 \mathrm{~km})$ com 15 municípios costeiros onde existe atividade pesqueira marinha. Quanto à aquicultura, ela foi introduzida na década de 1980, e desde entâo houve um grande desenvolvimento dessa atividade. Os primeiros passos, em 1987, foram dados de forma experimental no município de Piúma. Já nos últimos 17 anos, a atividade desenvolveu-se com fins comerciais em outros cinco municípios por meio de projetos com objetivos similares, mas com estratégias diferentes. Em face desse cenário, o presente estudo se mostrou oportuno e possibilita apontar um conjunto de informaçóes dos aspectos econômicos e sociais da pesca e da maricultura no Estado. Essas informaçóes podem contribuir para a definição de áreas estratégicas para o desenvolvimento das atividades de pesca e maricultura de forma sustentável, subsidiando a formulação de políticas de ordenamento do território e orientando as esferas governamentais na tomada de decisóes sobre políticas públicas. Como resultado, verificou-se que, além das potencialidades já existentes, há necessidade que o poder público dos municípios e do Estado, estabeleçam políticas de incentivo permanente a maricultura e pesca, como linhas de credito compatíveis com o perfil do pescador tradicional que precisa incrementar a sua renda, tendo em vista a pesca em declínio. Além disso, o acompanhamento das atividades é fundamental para o sucesso das mesmas. Fortalecer o associativismo, identificar gargalos e propor mudanças devem ser os principais itens de pauta da parceria maricultores/ pescadores e governo, propiciando o real desenvolvimento da atividade e a reduçáo das desigualdades sociais.

Palavras-chave: Aquicultura, Sustentabilidade, SIG, AHP.

\section{ABSTRACT}

Espirito Santo State (Southeast Brazil) has 15 municipalities in its coastal zone ( $140 \mathrm{~km}$ ). Aquaculture was introduced in the 1980s and during this period there was a major development of the activity. Since its beginnings in 1987 activities were experimental, as in Piuma District. In the last 17 years the activity has developed into commercial projects in five other cities. Although projects have similar goals, they diverge in strategies. This study points out a set of information regarding the economic and social aspects of fisheries and aquaculture in the state. Such information can contribute to the definition of strategic areas for the development of fisheries and aquaculture activities in a sustainable manner,

\footnotetext{
@ - Corresponding author

1 -Aqua-Ambiental-Aquicultura, Oceanografia e Meio Ambiente, Vitória, E.S., Brasil.e-mail: jboceano@gmail.com

2 - Universidade Federal do Espirito Santo, Centro Universitário Norte do Espirito Santo, UFES/CEUNES, Brasil. e-mail: aclpesca@yahoo.com.br

3 - Universidade Federal do Espirito Santo, Centro Universitário Norte do Espirito Santo, Departamento de Engenharias e Computação, Núcleo de Pesquisa em Gestão de Sistemas de Produção (NPGSP), Laboratório de Gestão Costeira - Aquicultura e Pesca (LGCap), São Mateus, ES, Brasil. e-mails: Boechat flaviopboechat@hotmail.com,Rodrigues-rafael.lima.rodrigues@hotmail.com, Freitas-rodrigorandow@ig.com.br
} 
supporting the formulation of public policies. Our main result was to identify the need for public institutions of the state and municipalities to establish incentive permanent policies for fishing and aquaculture, with lines of credit consistent with the profile of the traditional fisherman. Moreover, monitoring the activity will be fundamental for its success. Strengthening of the association fishers-government, identification of constrains and adaptation should be the main agenda to enable the development of fisheries/aquaculture sustainability and the reduction of social inequities.

Keywords: Aquaculture, Sustainability, GIS, AHP.

\section{INTRODUÇÁO}

O estado do Espírito Santo (Brasil) é possui uma linha de costa que, de acordo com o Ministério do Meio Ambiente (MMA, 1997), possui cerca de $410 \mathrm{~km}$ de extensão. Ao longo desse litoral existem 15 municípios costeiros onde existe atividade pesqueira marinha. Esta atividade é considerada basicamente artesanal por ser predominante o sistema de produção familiar e de subsistência, de parceria ou armadores. Constituem exceções as regióes central e sul, onde um pequeno número de empresas exerce a atividade de forma organizada, planejada e estrutura em relação à pesca. De acordo com o\$ III do Art. $2^{\circ}$ da Lei Federal no 11.959, "pesca: toda operação, açâo ou ato tendente a extrair, colher, apanhar, apreender ou capturar recursos pesqueiros" (D.O.U., 2009).

Segundo, Martins \& Doxsey (2006), a principal estratégia de pesca, em termos de frota e mão de obra envolvida, é a pesca de linha de mão, embora fraçóes importantes das pescarias empreguem redes de arrasto de fundo para captura de camarão e redes de espera para captura de peixes costeiros.

Com o objetivo de diagnosticar a situação da pesca no estado do Espírito Santo, vários estudos foram realizados desde a década de 1980 (Espírito Santo, 1976) e, mais recentemente (Centoducatte et al., 2005; Souza \& Oliveira, 2003; Monjardim, 2004; Pizzeta, 2004; PROMAR, 2005; Andrade, 2006; Martins \& Doxsey, 2006).

Souza \& Oliveira (2003), estabeleceram uma projeção da produção de pescado no Espírito Santo, em torno de 13.000 toneladas por ano, movimentando cerca de $\mathrm{R} \$ 130$ milhóes anualmente. Estimaram um total de 1.666 embarcações. Já Martins \& Doxsey (2006) encontraram uma produção anual em torno de 22.095 toneladas com estimativa de 6.733 pescadores trabalhando em 2.038 embarcaçóes. $\mathrm{O}$ fato deste volume ter sido maior para o ano de 2006 não representa um processo um crescimento, visto que a estimativa citada para 2006 considerou a pesca artesanal, que foi excluída do somatório de Souza \& Oliveira (2003).

De acordo com IBAMA (2008), no Espírito Santo o crescimento na produção de pescado foi de $16 \%$ em relação a 2005. Sendo que as espécies de peixes que mais contribuíram para esse acréscimo foram: cação $(63,8 \%)$, espadarte $(52,5 \%)$, dourado $(21,2 \%)$ e o badejo $(11,2 \%)$, todavia, a produção do peroá apresentou um decréscimo de 5,2\% em relação ao ano de 2006.

Quanto à aquicultura no Estado do Espírito Santo, ela foi introduzida na década de 1980, pelos esforços conjuntos das antigas Empresa Capixaba de Pesquisa Agropecuária (EMCAPA) e Empresa de Assistência Técnica e Extensão Rural do Espírito Santo (EMATER), as quais juntas constituem hoje o Instituto Capixaba de Pesquisa e Extensão Rural (INCAPER). Também houve uma participação notável do Instituto Brasileiro do Meio Ambiente e Recursos Naturais Renováveis (IBAMA). Essas instituições implantaram estaçóes de produção de alevinos de peixes e pós-larvas de camaróes de água doce que alavancaram o setor. Nesse período houve um grande desenvolvimento da atividade, chegando-se a 100 produtores envolvidos e uma área alagada em torno de 120 hectares (Oliveira et al., 2007).

A implantação da maricultura no Estado em 1987 foi de forma experimental no município de Piúma. Nos últimos 17 anos, a atividade foi implantada com fins comerciais em outros cinco municípios por meio de projetos com objetivos similares, mas de estratégias diferentes (Sodré et al., 2008). Atualmente, a maricultura apresenta grande potencial de desenvolvimento, porém os projetos existentes são extremamente tímidos. Até 2007 a mitilicultura (cultivo de mexilhóes) beneficiava 80 famílias no litoral sul, produzindo 40 toneladas de mexilhão limpo por ano, e estava representada pela Associação dos Maricultores de Anchieta (AMA) e a Associação de Maricultores de Guarapari. A ostreicultura (cultivo de ostras) envolvia 25 famílias e produzia uma média de 50.000 unidades por ano, sendo representada pela Associação dos Maricultores de Conceição da Barra e a Associação Aquícola de Guarapari. Ambas as atividades envolviam principalmente pescadores artesanais e catadores de mariscos (Oliveira et al., 2007).

Assim, em face desse cenário, o presente estudo se mostra oportuno e propiciará um conjunto de informaçóes dos aspectos econômicos e sociais inerentes a pesca e maricultura em nível estadual. A base dessas informaçóes contribuirá para a definição de áreas estratégicas para o desenvolvimento das atividades de pesca e maricultura de forma sustentável, subsidiando a formulação de políticas de ordenamento do território e orientando as esferas governamentais para tomada de decisóes em políticas públicas.

\section{MATERIAIS E MÉTODOS}

\section{1. Área de estudo}

Aárea de abrangência do presente estudo são 15 municípios costeiros do estado do Espírito Santo (ES) (20¹9'40.12" S; $\left.40^{\circ} 16^{\prime} 14.48^{\prime \prime} \mathrm{O}\right)$. Para uma melhor compreensão dividiuse os mesmos em três regióes, definidas de acordo com as características intrínsecas de desenvolvimento, proximidade, econômicas, sociais e culturais de cada local e sua posição geográfica no estado. As regióes são: Região norte (Conceição da Barra, São Mateus e Linhares - limítrofe do Rio Doce); Regiāo Centro (Aracruz, Fundão, Serra, Vitória, Cariacica 
e Vila Velha) e; Região Sul (Guarapari, Anchieta, Piúma, Itapemirim, Marataízes e Presidente Kennedy). Todos os municípios fazem parte da regiáo avaliada para o componente de potencialidade para implantação do cultivo de peixes.

\subsection{Metodologia Aplicada}

Primeiramente uma revisão bibliográfica foi estruturada com finalidade de definir o real posicionamento do tema no tempo e no espaço, além de analisar as diversas metodologias empregadas na identificação e descrição dos diferentes tipos de entraves e potencialidades da atividade de piscicultura marinha e estuarina observadas. Buscaram-se, assim somente dados bibliográficos secundários oficiais e publicados disponíveis.

Quanto à metodologia adotada para integração e análise dos dados que constituem a potencialidade social e econômica nos municípios costeiros no Estado do Espírito Santo, referente à potencialidade de implantaçáo de empreendimentos piscícolas, utilizou-se a ferramenta Analytic Hierarchy Process (AHP) (Saaty, 2008) para hierarquização dos critérios mais importantes para a confecção de uma matriz de comparação par a par. Assim também utilizado por (Terfai \& Schrimpf, 1998). A partir do agrupamento, análise e hierarquização dos dados coletados, juntamente com o intuito de proporcionar uma melhor visualização e integração dos resultados obtidos, utilizou-se um Sistema de Informação Geográfica (SIG).

Tratando-se do método AHP, ele se divide em definir o problema, estruturar a hierarquia de decisão, construçâo das matrizes par a par, sintetizar os dados. Com isso pretendeuse definir o objetivo principal do estudo, buscando apontar as fragilidades e as potencialidades dos municípios perante piscicultura marinha e estuarina. Sendo que a estrutura hierárquica foi construída com o objetivo principal no topo, seguida de níveis intermediários (indicadores) para um nível mais baixo (municípios).
Com a compilação dos dados estruturados, foram selecionados critérios específicos, baseados em conceitos e modelos retirados da bibliografia e a experiência do grupo. A partir da etapa acima descrita, inicia-se a construção da matriz e comparaçôes par a par. Para sua confecçáo foi necessário o julgamento entre os critérios pelos autores, considerando a Escala Fundamental de Saaty (2008) (Tabela 1).

Utilizando a matriz de importância relativa (Tabela 1), foram atribuídos pesos diferentes para cada critério utilizado. Os pesos atribuídos variaram de 1 a 9 (utilizando apenas os números impares), de acordo com o grau de importância de um comparado com o outro. Por exemplo, o número de pescadores/maricultores quando comparado com o número de barcos teve um grau de importância 3 na escala fundamental de Saaty (2008) (isso significa que o número de pescadores/maricultores tem importância moderada quando comparado com o número de embarcações), já quando comparado com a distância da capital Vitória teve grau de importância 9 (isso significa que o número de pescadores/ maricultores é extremamente mais importante que a distância da capital Vitória). Com o resultado da pontuaçáo derivada da Matriz de Importância Relativa, foram plotadas cartas, para cada indicador, para cada fator condicionante e para cada critério do componente, utilizando-se o programa ArcGIS 9.3.

Devido à natureza espacial das informaçóes e à necessidade do produto final ser georreferenciado, buscouse uma forma de apresentaçáo específica, com que cada categoria correspondesse a um número e uma cor específica, procedimento que diferenciaria um município do outro. As cores utilizadas nas cartas são equivalentes às categorias e aos valores de potencialidade utilizados para categorizar os municípios, quando temos um ponto de partida muito precário, foi sinalizado com a cor vermelha, quando teve precário, amarelo, pouco favorável, verde, favorável, azul claro e quando teve o ponto de partida muito favorável teve a cor azul.

Tabela 1. Escala fundamental de números absolutos referentes à importância relativa entre os critérios (Adaptação de Saaty, 2008). Table 1. Fundamental scale of absolute numbers regarding the relative importance of the criteria (Adapted from Saaty, 2008).

\begin{tabular}{ccc}
$\begin{array}{c}\text { Intensidade de } \\
\text { importância }\end{array}$ & Definiçáo & Contextualizaçáo \\
\hline 1 & Igual importância & Os dois critérios contribuem igualmente para vulnerabilidade ou \\
potencialidade
\end{tabular}


A partir disso, normalizou-se cada indicador em um número mínimo (1) e um número máximo (5), com três intervalos entre eles (2, 3 e 4). Dessa forma, cada indicador se enquadrou em uma dessas cinco categorias, sendo que os dados relativizados gerados apontaram em que ponto de partida, menos ou mais favorável, cada município se encontra comparativamente entre os demais.

Sendo que, primeiramente, objetivando um melhor entendimento, buscou-se agrupar em um único banco de dados todos os indicadores (critérios) que caracterizavam/ refletiam o estágio de desenvolvimento da atividade de Maricultura e Pesca no Estado (Tabela 2). Ressaltasse também que as informaçóes foram analisadas em conjunto e não de forma individual, uma vez que, as articulaçóes entre elas são essenciais para demonstrar a configuração de uma dada realidade municipal e estadual.

Tabela 2. Banco de dados dos critérios/indicadores por unidade e por valores normalizados de 1 a 5 .

Table 2. Database criteria / indicators per unit and normalized values of 1 to 5.

\begin{tabular}{|c|c|c|c|c|c|c|c|c|c|c|c|c|c|c|c|c|}
\hline $\begin{array}{l}\text { Critério/ } \\
\text { Indicador }\end{array}$ & Código & 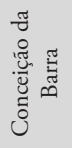 & 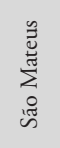 & 总 & 芯 & 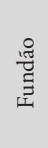 & 芯 & 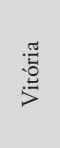 & 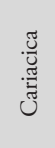 & $\frac{\pi}{\frac{\pi}{5}}$ & 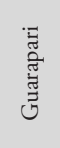 & 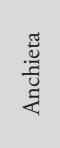 & $\mathfrak{\Xi}^{\pi}$ & 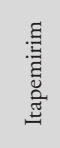 & 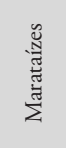 & 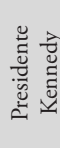 \\
\hline \multirow{2}{*}{$\begin{array}{c}\text { Locais de } \\
\text { desembarque }\end{array}$} & \multirow{2}{*}{ DESEMBARQUE } & 2 & 5 & 3 & 3 & 1 & 3 & 3 & 0 & 6 & 5 & 5 & 1 & 3 & 4 & 2 \\
\hline & & 2 & 4 & 3 & 3 & 2 & 3 & 3 & 1 & 5 & 4 & 4 & 2 & 3 & 3 & 2 \\
\hline \multirow{2}{*}{ Fábrica de gelo } & \multirow{2}{*}{ FAB_GELO } & 3 & 0 & 0 & 2 & 0 & 2 & 2 & 0 & 3 & 4 & 2 & 8 & 4 & 4 & 0 \\
\hline & & 3 & 1 & 1 & 2 & 1 & 2 & 2 & 1 & 3 & 4 & 2 & 5 & 4 & 4 & 1 \\
\hline \multirow{2}{*}{$\begin{array}{l}\text { Câmaras } \\
\text { frigoríficas }\end{array}$} & \multirow{2}{*}{ CAM_FRIGOR } & 1 & 0 & 0 & 4 & 0 & 1 & 2 & 0 & 1 & 2 & 1 & 3 & 2 & 2 & 0 \\
\hline & & 2 & 1 & 1 & 5 & 1 & 2 & 3 & 1 & 2 & 3 & 2 & 4 & 3 & 3 & 1 \\
\hline \multirow{2}{*}{ Câmaras frias } & \multirow{2}{*}{ CAM_FRIA } & 6 & 0 & 0 & 0 & 0 & 0 & 1 & 0 & 1 & 7 & 2 & 10 & 7 & 6 & 0 \\
\hline & & 3 & 1 & 1 & 1 & 1 & 1 & 2 & 1 & 2 & 4 & 2 & 5 & 4 & 3 & 1 \\
\hline \multirow{2}{*}{ Peixarias } & \multirow{2}{*}{ PEIXARIAS } & 52 & 8 & 15 & 12 & 3 & 22 & 38 & 1 & 18 & 43 & 15 & 8 & 14 & 28 & 0 \\
\hline & & 5 & 2 & 3 & 2 & 2 & 3 & 4 & 2 & 3 & 4 & 3 & 2 & 3 & 4 & 1 \\
\hline \multirow{2}{*}{$\begin{array}{c}\text { Unidades de } \\
\text { Beneficiamento }\end{array}$} & \multirow{2}{*}{ UNI_BEN } & 2 & 0 & 0 & 2 & 0 & 0 & 1 & 0 & 0 & 1 & 1 & 1 & 2 & 1 & 0 \\
\hline & & 5 & 1 & 1 & 5 & 1 & 1 & 3 & 1 & 1 & 3 & 3 & 3 & 5 & 3 & 1 \\
\hline \multirow[b]{2}{*}{ Estaleiro } & \multirow[b]{2}{*}{ ESTALEIROS } & 7 & 6 & 8 & 6 & 0 & 1 & 7 & 0 & 8 & 2 & 6 & 5 & 4 & 20 & 2 \\
\hline & & 4 & 3 & 4 & 3 & 1 & 2 & 4 & 1 & 4 & 2 & 3 & 3 & 3 & 5 & 2 \\
\hline \multirow{2}{*}{$\begin{array}{l}\text { Lojas de material } \\
\text { de pesca }\end{array}$} & \multirow{2}{*}{ LOJAS } & 3 & 0 & 0 & 2 & 0 & 0 & 5 & 0 & 1 & 5 & 3 & 3 & 5 & 4 & 0 \\
\hline & & 3 & 1 & 1 & 3 & 1 & 1 & 5 & 1 & 2 & 5 & 3 & 3 & 5 & 4 & 1 \\
\hline \multirow{2}{*}{$\begin{array}{l}\text { Embarcaçôes } \\
\text { pesqueiras }\end{array}$} & \multirow{2}{*}{ BARCOS } & 123 & 49 & 24 & 70 & 4 & 58 & 181 & 0 & 110 & 256 & 111 & 170 & 297 & 164 & 2 \\
\hline & & 3 & 2 & 2 & 2 & 2 & 2 & 3 & 1 & 3 & 4 & 3 & 3 & 5 & 3 & 2 \\
\hline \multirow{2}{*}{$\begin{array}{l}\text { Cultivos por } \\
\text { município }\end{array}$} & \multirow{2}{*}{ CULTIVO } & 1 & 1 & 0 & 0 & 0 & 0 & 0 & 0 & 0 & 2 & 2 & 1 & 0 & 1 & 0 \\
\hline & & 3 & 3 & 1 & 1 & 1 & 1 & 1 & 1 & 1 & 5 & 5 & 3 & 1 & 3 & 1 \\
\hline \multirow{2}{*}{$\begin{array}{l}\text { Laboratórios de } \\
\text { aquicultura }\end{array}$} & \multirow{2}{*}{$\mathrm{LAB}$} & 0 & 1 & 1 & 1 & 0 & 0 & 0 & 1 & 0 & 0 & 0 & 0 & 0 & 0 & 0 \\
\hline & & 1 & 5 & 5 & 5 & 1 & 1 & 1 & 5 & 1 & 1 & 1 & 1 & 1 & 1 & 1 \\
\hline \multirow{2}{*}{$\begin{array}{l}\text { Pescadores e } \\
\text { maricultores }\end{array}$} & \multirow{2}{*}{$\begin{array}{l}\text { PESCADOR_ } \\
\text { MARICULTOR }\end{array}$} & 620 & 120 & 185 & 206 & 24 & 401 & 920 & 268 & 508 & 772 & 541 & 500 & 2305 & 1385 & 28 \\
\hline & & 3 & 2 & 2 & 3 & 1 & 3 & 4 & 3 & 3 & 3 & 3 & 3 & 5 & 4 & 1 \\
\hline \multirow{2}{*}{$\begin{array}{c}\text { Ass. de } \\
\text { Pescadores / } \\
\text { Maricultores }\end{array}$} & \multirow{2}{*}{$\begin{array}{l}\text { ASSOPESCA } \\
\text { ASSOMAR }\end{array}$} & 2 & 4 & 2 & 0 & 0 & 3 & 1 & 1 & 2 & 3 & 2 & 0 & 1 & 1 & 2 \\
\hline & & 3 & 5 & 3 & 1 & 1 & 4 & 2 & 2 & 3 & 4 & 3 & 1 & 2 & 2 & 3 \\
\hline Distância de & & 251 & 217 & 134 & 82 & 53 & 25 & 0 & 17 & 8 & 51 & 78 & 89 & 120 & 116 & 158 \\
\hline Vitória & DISI_VII & & 2 & 2 & 3 & 3 & 4 & 5 & 4 & 4 & 4 & 3 & 3 & 2 & 3 & 2 \\
\hline
\end{tabular}




\section{RESULTADOS E DISCUSSÓES}

Assim, a cada categoria corresponde um número e uma cor, apresentada nas cartas, que diferencia um município do outro. As cores utilizadas nas cartas são equivalentes às categorias e aos valores de potencialidade utilizados para categorizar os municípios. Em relação à divisão dos municípios em categorias, ela foi feita com base nas potencialidades apresentadas por cada um. Demonstrado que os municípios partem de pontos diferenciados de condições produtivas, naturais, humanas e institucionais. Portanto, o poder público deve tratar cada município de forma individual, observando sempre as particularidades locais ou regionais.

Assim, com base nessa categorização, o Governo do Estado do Espírito Santo poderá tomar decisóes estratégicas, definir metas, recursos, prazos e elaborar planejamento para municípios específicos ou regiōes inteiras de forma mais adequada a cada realidade.

A partir da divisão por regiôes, os dados do Componente de Potencialidade Social da Pesca e Maricultura foram representados em um mapa final (Figura 1).

Assim, ao analisarmos os resultados obtidos dos municípios por região, no sul (Figura1), destacaram-se os municípios de Itapemirim e Marataízes. Esses, considerados como ponto de partida em condiçóes muito favoráveis ao desenvolvimento da maricultura e pesca. Isso se deve a uma junção de fatores. Isso devido aos dois municípios juntos empregarem 3.690 pescadores/maricultores $(41,01 \%$ do total de pescadores/maricultores do Estado). Sendo que os dois detêm ainda, cerca de 30\% da frota estadual, com 461 embarcaçôes.

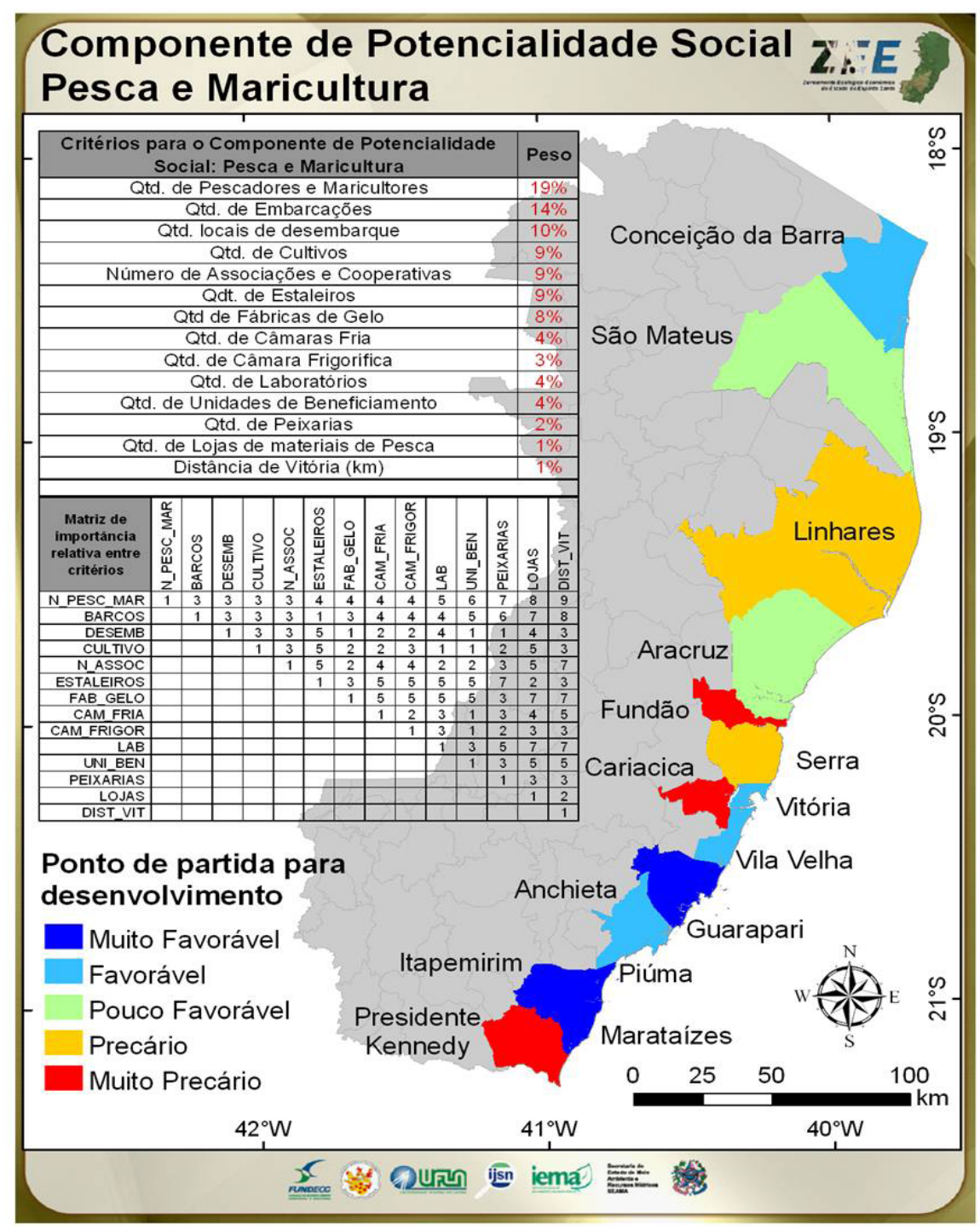

Figura1. Potencialidade social e econômica dos municípios costeiros, segundo os componentes: Pesca e Maricultura.

Figure1. Social and economic potential of coastal municipalities, according to the components: Fishing and sea culture. 
$\mathrm{Na}$ região Metropolitana de Vitória, o município de Guarapari é o único com ponto de partida em condiçóes muito favoráveis. $\mathrm{Na}$ pesca o município se destaca por possuir o segundo maior número de embarcaçóes no Estado, ficando atrás apenas de Itapemirim, e o quarto em número de pescadores/maricultores. Vitória e Vila Velha vêm logo em seguida, possuindo o terceiro e o sétimo maior número de pescadores/maricultores respectivamente. Vitória detém o terceiro maior número de embarcaçóes do Estado.

A região Norte variou do ponto de partida precário (Linhares), a pouco favorável, (Aracruz). Considerando a regionalização, o município de Aracruz detém 206 pescadores/maricultores e 70 embarcaçóes, já o município de Linhares possui aproximadamente 185 pescadores/ maricultores e 24 embarcaçóes. No Extremo Norte capixaba, quem se destaca é o município de Conceição da Barra, com o quinto maior número de pescadores/maricultores do Estado (630 trabalhadores) e a sexta maior frota pesqueira (123 embarcaçôes).

\section{CONSIDERAÇÓES FINAIS}

Considerando a falta de material bibliográfico publicado com informaçóes mais detalhadas, recentes e disponíveis para a confecção desse estudo, fica evidente a falta de foco na maricultura e pesca no estado. Pesquisas em nível acadêmico e governamental que possibilitem o desenvolvimento das atividades pesqueiras e aquícolas são bastante valiosas, pois propiciam visualizar os diferentes graus de desenvolvimento dos municípios, quanto à infraestrutura urbana, social e econômico/financeira, permitindo ao final das análises, concluir que o estado possui um papel fundamental na diminuição das desigualdades apontadas, contribuindo junto às prefeituras locais para a implantação, desenvolvimento e a sustentabilidade das atividades.

Assim, é de suma necessidade que o poder público dos municípios e/ou estado, estabeleça políticas de incentivo permanente a maricultura e pesca, com linhas de credito compatíveis principalmente ao perfil do pescador tradicional que precisa incrementar a sua renda, tendo em vista a pesca em declínio.

Além disso, o acompanhamento da atividade e fundamental para o sucesso da mesma. Fortalecer o associativismo, identificar gargalos e propor mudanças devem ser os principais itens de pauta da associação entre maricultores/pescadores e governo, propiciando o real desenvolvimento da atividade e a redução das desigualdades sociais.

\section{BIBLIOGRAFIA}

Andrade, H.K. (2006) - Aquicultura Capixaba: da produção ao mercado. 232p., ECOS - Instituto de Pesquisa e Desenvolvimento Sócio-ambiental, Vitória, ES, Brasil.

Centoducatte, J.G.; Teixeira, J.B.; Barroso, G.F. (2005) Macrodiagnóstico da Pesca Marítima do Estado do Espirito Santo. Relatório Técnico, 68p., Fundação PROMAR / SEAG - Secretaria de Agricultura, Abastecimento e Pesca. Vitória, ES, Brasil.

DOU (2009) - Lei no 11.959, de 29 de junho de 2009. Dispóe sobre a Politica Nacional de Desenvolvimento
Sustentável da Aquicultura e da Pesca, regula as atividades pesqueiras, revoga a Lei $n^{\circ} 7.679$, de 23 de novembro de 1988, e dispositivos do Decreto-Lei $n^{\circ} 221$, de 28 de fevereiro de 1967, e dá outras providências. Publicado no DOU de 30.6.2009, p.1, Brasília, DF, Brasil. Disponível em http://www.planalto.gov.br/ccivil_03/_Ato20072010/2009/Lei/L11959.htm

IBAMA (2008) - Estatística da Pesca 2006 BRASIL. Grandes regiōes e unidades da Federação. 174p., IBAMA - Instituto Brasileiro do Meio Ambiente e dos Recursos Naturais Renováveis, Brasil. ISBN: 978-8573002768.

Martins, A.S.; Doxsey, J.R. (2006) - Diagnóstico da pesca no litoral do estado do Espírito Santo. In: V.J. Isaac, A.S. Martins, M. Haimovici, J.M. Andriguetto Filho, (org), A pesca marinha e estuarina do Brasil no início do século XXI: recursos tecnologias, aspectos socioeconômicos e institucionais, pp.93-116, Editora Universitária UFPA, Belém, PA, Brasil. ISBN: 85-24703458.

MMA (1997) - Espirito Santo. In: R.E.C. Gualda (coord.), Conservação Ambiental no Brasil. Relatório de Atividades (1991/96), Proteçáo de Ecossistemas, 3 - Zona Costeira [on-line], MMA - Ministério do Meio Ambiente, Brasília, DF, Brasil. Disponivel em http://www.mma.gov.br/port/ se/pnma/ecos16.html .

Monjardim, C. (2004) - Avaliação Multidimensional dos Sistemas Pesqueiros da Região Central e Norte do Espirito Santo, Brasil, e seus Indicadores de Sustentabilidade. 71p., Dissertação de Monografia (Graduação em Oceanografia), Departamento de Ecologia e Recursos Naturais, Universidade Federal do Espírito Santo, Vitória, ES, Brasil. Disponível em http://www.dern.ufes.br/oceano/ link/monografias/2003/monog2_2003.pdf

Oliveira, J.C.; Souza, A.C.C.; Valle, J. de B. (coord.) (2007) - Plano Estratégico de Desenvolvimento da Agricultura Capixaba. 21p., Governo do Estado do Espírito Santo, Vitória, Brasil. http://www.seag.es.gov.br/pedeag/setores/ aquicultura.pdf

Pizetta, G.T. (2004) - Avaliação Multidimensional dos Sistemas Pesqueiros da Região Sul do Espirito Santo, Brasil, e seus Indicadores de Sustentabilidade. 72p., Dissertação de Monografia (Graduação em Oceanografia), Departamento de Ecologia e Recursos Naturais, Universidade Federal do Espírito Santo, Vitória, ES, Brasil. Disponível em http:// www.dern.ufes.br/oceano/link/monografias/2003/ monog4_2003.pdf

PROMAR (2005) - Macrodiagnóstico do potencial do ES para implantação de projetos de aqüicultura de águas interiores, estuarinas e marinhas. Relatório Técnico, 32p., Fundação Promar, Brasil. Disponível em http://www.fundagres. org.br/macrodiagnostico/relatorio_macro.pdf

Saaty, T.L. (2008) - Decision making with the analytic hierarchy process. International Journal of Services Sciences (IJSSCI), 1(1):83-98. DOI: 10.1504/ IJSSCI.2008.017590

Espírito Santo Secretaria do Estado da Agricultura (1976) Diagnóstico da pesca artesanal do Espirito Santo. Secretária do Estado da Agricultura, Brasil. In: http://www.ijsn. es.gov.br/Busca/?module=Record\&id $=11122$.

Sodré, F.N.G.A.S.; Freitas, R.R.; Rezende, V.L.F.M. (2008) - Histórico e desenvolvimento da maricultura no Estado 
do Espirito Santo. Revista Brasileira de Agroecologia (ISSN: 1980-9735), 3(3):36-46, Cruz Alta, RS, Brasil. Disponível em http://www.aba-agroecologia.org.br/ojs2/ index.php/rbagroecologia/article/view/7543

Souza, A.C.C.; Oliveira, J.C. (2003) - Plano Estratégico da Agricultura Capixaba: Estudo Temático Pesca. v.14, Vitória, Brasil. Secretaria do Meio Ambiento - 2012:
Ano Internacional da Energia Sustentável para Todos, Governo do Estado de São Paulo, Secretaria do Meio Ambiente, São Paulo, Brasil.

Terfai, L.; Schrimpf, W. (1998) - Development of an Aquaculture Information System for shrimp farming in warm water environment. 4th African Conference on Research in Computer Science, Dakar Senegal. 